\title{
Hydrodynamics of Clumpy Flows: Application to the PNe
}

\author{
A.Y. Poludnenko, A. Frank, \& E.G. Blackman \\ Department of Physics and Astronomy, \\ University of Rochester, Rochester, NY 14627-0171
}

\begin{abstract}
Many astrophysical flows occur in inhomogeneous media. We present results of a hydrodynamic numerical study of the interaction of a steady, planar shock/supersonic postshock flow with a system of embedded cylindrical clouds in a two-dimensional geometry and describe an analytical model of the evolution of such systems. Finally we discuss mass-loading in such systems and the applicability of the results to the planetary nebulae (PNe).

The most recent results and animations of the numerical experiments described in this paper can be found at www.pas.rochester.edu $/{ }^{\sim}$ wma.
\end{abstract}

\section{Introduction}

Mass outflows play a critical role in many astrophysical systems. Virtually all studies of mass outflows to date have focused on flows in homogeneous media. The typical astrophysical medium, however, is inhomogeneous with "clumps" or "clouds" arising on a variety of scales. Whatever the origin of the clumps, their presence can introduce not only quantitative but also qualitative changes to the overall dynamics of the flow. Planetary nebulae are an important astrophysical example of flows where the presence of inhomogeneities may play a conspicuous role in defining nebular dynamics and morphology.

In planetary nebulae the physical mechanism responsible for clump destruction and mixing remains unclear. The mixing of clump material with the ambient flow in planetary nebulae, such as NGC 7293 (Helix) or NGC 2392 (Eskimo), may occur via hydrodynamic ablation, photoevaporation, or the combination of the two. However, in order to gain basic understanding of the underlying physics, we focused on the case of purely hydrodynamic interaction.

\section{Analytical and Numerical Results}

We present results of a numerical study of the interaction of a strong, steady, planar shock with a system of clumps in a two-dimensional geometry (Poludnenko et al. 2002). Our results are applicable to shocks with Mach numbers $3<M_{S}<1000$. The range of applicable cloud/ambient density contrast values is $10-1000$. We neglect any radiative losses, heat conduction, and gravity.

Our numerical code uses an adaptive mesh refinement algorithm for the equations of gas dynamics in the AMRCLAW implementation (Berger \& LeV- 
eque 1998). It allows us to achieve sufficiently high resolution both at the largest and the smallest scales.

We find four major phases of the cloud evolution due to the interaction of the global shock and postshock flow with a system of clouds. We describe a simple model for the cloud acceleration during the first three phases, i.e. prior to its destruction, and derive expressions for the cloud velocity and displacement. The maximum cloud displacement (prior to its destruction) does not exceed 3.5 initial maximum cloud radii. The maximum cloud velocity is not more than $10 \%$ of the global shock velocity (Poludnenko et al. 2002).

Detailed analysis of the simulations shows that interaction of embedded inhomogeneities with the shock/postshock wind depends primarily on the thickness of the cloud layer and arrangement of the clouds in the layer. The total cloud mass and the total number of individual clouds is a far less significant factor. We define two classes of cloud distributions: thin and thick layers. We define the critical cloud separation both along and perpendicular to the direction of the flow distinguishing between interacting and noninteracting regimes of cloud evolution. The key parameters determining the type of the cloud system evolution are the critical cloud separation transverse to the flow $d_{\text {crit }}$, and the cloud destruction length $L_{C D}$. For a given astrophysical situation our results indicate that one might determine, either from observations or from theoretical analysis, the values of $d_{c r i t}$ and $L_{C D}$ and, thereby, describe the future evolution of a given system (Poludnenko et al. 2002).

One of the most important dynamical effects of the presence of inhomogeneities, previously suggested by Dyson \& Hartquist (1992), is mass-loading. We have examined the effect of mass-loading in our simulations. Our principal conclusion is that mass-loading is not significant in regimes studied in this work. This is due to short survival times of clouds under such conditions as well as to the very low mass loss rates of the clouds even during the times prior to their destruction. Mass loading may well be important in higher density clouds.

The developed general formalism can be applied to the description of the evolution of the clumpy shells in the planetary nebulae, e.g. NGC 2392 (Eskimo), and the influence of such shells on the global properties of the planetary nebulae.

Acknowledgments. AYP and AF acknowledge support from NSF grant AST-9702484 and NASA grant NAG5-8428. EGB acknowledges support from DOE grant DE-FG02-00ER5460. The authors acknowledge DOE support from the Laboratory for Laser Energetics.

\section{References}

Berger, M.J. \& LeVeque, R.J. 1998, SIAM J. Numer. Anal., 35, 2298

Dyson, J.E. \& Hartquist, T.W. 1992, ApL, 28, 301

Poludnenko, A. Y., Frank, A., Blackman, E. G. 2002, ApJ, 576, 832 